\title{
Exploration of Macro-Micro Biomarkers for Dampness-Heat Syndrome Differentiation in Different Diseases
}

\author{
Jianye Dai, ${ }^{1}$ Shujun Sun, ${ }^{1}$ Jinghua Peng, ${ }^{2}$ Huijuan Cao, ${ }^{1}$ Ningning Zheng, \\ Junwei Fang, ${ }^{1}$ Qianhua Li, ${ }^{1}$ Jian Jiang, ${ }^{2}$ Yongyu Zhang, ${ }^{1}$ and Yiyang $\mathrm{Hu}^{2}$ \\ ${ }^{1}$ Center for Traditional Chinese Medicine and Systems Biology, Shanghai University of Traditional Chinese Medicine, \\ Shanghai 201203, China \\ ${ }^{2}$ Institute of Liver Diseases, Shuguang Hospital, Key Laboratory of Liver and Kidney Diseases of Ministry of Education, \\ Shanghai University of Traditional Chinese Medicine, Shanghai 201203, China
}

Correspondence should be addressed to Yongyu Zhang; dryyz@sina.com and Yiyang Hu; yyhuliver@163.com

Received 3 January 2013; Revised 6 February 2013; Accepted 17 February 2013

Academic Editor: Wei Jia

Copyright (C) 2013 Jianye Dai et al. This is an open access article distributed under the Creative Commons Attribution License, which permits unrestricted use, distribution, and reproduction in any medium, provided the original work is properly cited.

\begin{abstract}
Increased attention is being paid to traditional Chinese medicine (TCM) as a complementary and alternative medicine to provide an effective approach for personalized diagnosis and clinical treatment. TMC performs treatment based on differentiation of TCM syndrome (ZHENG), which may identify special phenotypes by symptoms and signs of patients even if they are in different diseases. There has, however, been skepticism and criticism because syndrome classification only depends on observation, knowledge, and clinical experience of TCM practitioners, which lacks objectivity and repeatability. In order to transform syndrome classification into mainstream medicine, we introduce a macro-micro approach that combines symptoms, clinical indicators, and metabolites. The present paper explores the macro-micro biomarkers of dampness-heat syndrome in chronic hepatitis B and nonalcoholic fatty liver patients, which could provide the basis for developing a possible population-screening tool for selecting target individuals and creating an evaluation index for personalized treatment.
\end{abstract}

\section{Introduction}

Chronic hepatitis $\mathrm{B}(\mathrm{CHB})$ and nonalcoholic fatty liver disease (NFL) are two common diseases occurring throughout the world that have continuously increasing morbidity [1]. It is worth noting that $12.1 \%$ [2] and $37.1 \%$ [3] of patients with $\mathrm{CHB}$ and NFL, respectively, exhibit the same symptoms (e.g., yellow and slimy fur), which are characteristics of dampnessheat syndrome (DH) in traditional Chinese medicine (TCM). Although CHB and NFL have different etiologies in Mainstream Medicine, TCM practitioners may perform the same treatment for these patients.

Actually, different diseases may be treated similarly in TCM particularly when the same syndrome appears in these diseases [4]. In this respect, syndrome differentiation and treatment (bian zheng lun zhi) may provide some new revelations to modern personalized medicine [5-7]. Syndrome differentiation is still debated, because it depends on clinical observation and TCM practitioners' experiences, which are thought to be subjective and unrepeatable. The success of personalized medicine relies on having accurate diagnostic tests that identify those patients who can benefit from targeted therapies [8]; thus, the ability to achieve objectivity and repeatability in TCM diagnosis would provide a greatly needed breakthrough.

Recently, researchers and scientists of TCM have explored incorporating several potentially beneficial methods, including, for example, physiology and biochemistry [9], molecular biology [10], and tongue image digitization [11, 12]. However, the classifications have been less than satisfactory. The main reason might be that these methods only focus on one or several indicators and thus cannot generalize the entire state of the syndrome. We therefore conceived the possibility of a macro-micro approach that includes a combination of metabolites, symptoms, and clinical indicators. Clinical manifestations are the macroeconomic performance, and metabolic molecules and indicators are microscopic. To serve in TCM diagnosis and treatment, here we report our findings 
from a case study that allowed us to preliminarily explore the macro-micro biomarkers of $\mathrm{DH}$ in $\mathrm{CHB}$ and NFL patients.

\section{Experimental}

2.1. Subjects and Experiment Design. Twenty healthy volunteers and 115 patients (60 patients for training and another 55 patients for testing) of dampness-heat syndrome chronic hepatitis $\mathrm{B}$ (DHHB), nondampness-heat syndrome chronic hepatitis B (NDHHB), and dampness-heat syndrome nonalcoholic fatty liver (DHFL) were enrolled in the study. The clinical study was approved by the local ethics committee and was performed in accordance with the principals contained in the Declaration of Helsinki. All individuals provided informed consent before inclusion into the study. Diagnostic standard of $\mathrm{HB}$ and FL patients was referred to "the guideline of prevention and treatment for chronic hepatitis B" [13] and "guidelines for management of nonalcoholic fatty liver disease: an updated and revised edition" [14]. Cases meeting the diagnostic criteria for chronic hepatitis B and nonalcoholic fatty liver, respectively, at 18-65 (39.9 mean \pm 13.5 std. dev.) years of age who signed the informed consent form were included in the study. Individuals were excluded from the study if they met any of the following criteria. (1) Cases complicated with other hepatotropic virus hepatitis and alcoholic fatty liver. (2) Chronic severe hepatitis. (3) HB and FL patients associated with serious primary disease of heart, kidney, lung, endocrine, blood, metabolic and gastrointestinal, or psychotic patients. (4) Pregnant or lactating women. A junior medical physician made the initial diagnosis and recorded the information of four traditional examinations accurately and completely. Three more senior physician (either chief or deputy physicians) subsequently confirmed the initial diagnosis by the records and gave the hierarchical results of typical degree. Only those cases that were identified as classical DH patients by both the junior and the senior physicians were included in the study to guarantee the correctness of ZHENG differentiation.

2.2. Chemicals and Drugs. N,O-bis (trimethylsilyl) trifluoroacetamide (BSTFA with 1\% TMCS) and urease were purchased from Sigma-Aldrich Co. LLC (USA). Methoxyamine hydrochloride, methanol, ethanol, myristic acid, chloroform and pyridine were purchased from China National Pharmaceutical Group Corporation (Shanghai, China).

2.3. Sample Collection and Preparation. A complete physical examination was given, and the health condition was recorded on a scale including the information obtained through four traditional examinations: looking, listening and smelling, asking, and touching when the patient entered the study. Seventy-one clinical indicators and 115 contents from the four methods of examinations were acquired for the basic information.

Urine samples were collected from all subjects and were stored at $-80^{\circ} \mathrm{C}$ until GC-MS assay. All urine samples were thawed in an ice water bath and vortex-mixed before analysis. Each $1 \mathrm{~mL}$ aliquot of standard mixture or urine sample was placed into a screw top tube, samples were centrifuged for

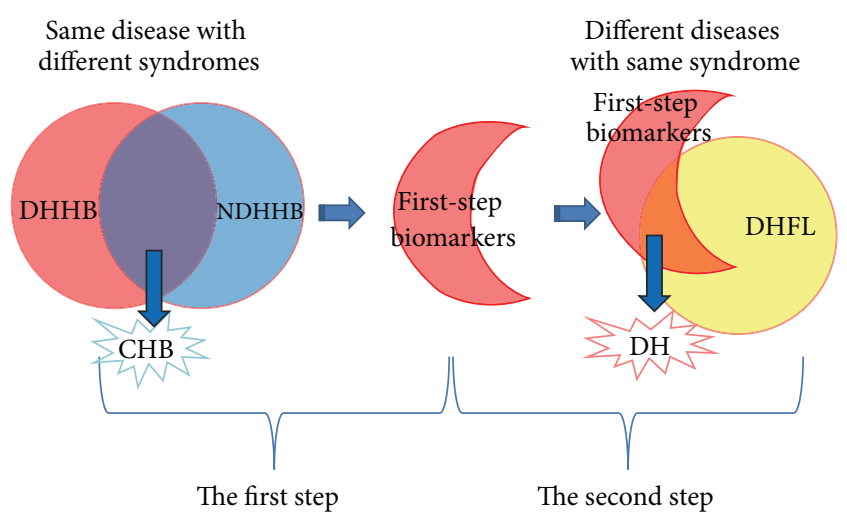

FIGURE 1: Schematic diagram of research approach for selection of $\mathrm{DH}$.

$10 \mathrm{~min}$ at $(12,000 \mathrm{rpm})$, and $150 \mu \mathrm{L}$ supernatants were then transferred into clean screw top tubes. After adding $70 \mu \mathrm{L}$ of urease $(4 \mathrm{mg} / \mathrm{mL})$ and vortex-mixing for $30 \mathrm{~s}$, samples were conditioned at $37^{\circ} \mathrm{C}$ for $15 \mathrm{~min}$ to remove the urea. After the addition of $800 \mu \mathrm{L}$ methanol and $10 \mu \mathrm{L}$ of myristic acid in methanol $(1 \mathrm{mg} / \mathrm{mL})$ and mixing for $1 \mathrm{~min}$, the solution was centrifuged at $13,000 \mathrm{rpm}$ for $10 \mathrm{~min}$. A $200 \mu \mathrm{L}$ aliquot of supernatant was then transferred into a GC vial and evaporated to dryness under $\mathrm{N}_{2}$ at $30^{\circ} \mathrm{C}$. Fifty $\mu \mathrm{L}$ of methoxyamine in pyridine $(15 \mathrm{mg} / \mathrm{mL})$ was added to the $\mathrm{GC}$ vial, and vortex-mixed for $1 \mathrm{~min}$, and the methoximation reaction was carried out for $90 \mathrm{~min}$ rocking in a shaker at $30^{\circ} \mathrm{C}$, then $50 \mu \mathrm{L}$ of BSTFA plus $1 \%$ TMCS was added to the samples for trimethylsilylation for another $1 \mathrm{~h}$ at $70^{\circ} \mathrm{C}$. In the final step, $30 \mu \mathrm{L}$ of heptane was added to the GC vial, and the solution was analyzed utilizing GC-MS after vortex for $30 \mathrm{~s}$.

2.4. Data Acquisition. All GC-MS analyses were performed by a mass spectrometer 5975B (Agilent technologies, USA) coupled to an Agilent 6890 (Agilent technologies, USA) gas chromatography instrument. In the gas chromatographic system, a catabletary column (Agilent J\&W DB-5 ms Ultra Inert $30 \mathrm{~m} \times 0.25 \mathrm{~mm}$, film thickness $0.25 \mu \mathrm{m}$ ) was used. Helium carrier gas was used at a constant flow rate of $1.0 \mathrm{~mL} * \mathrm{~min}^{-1}$. One $\mu \mathrm{L}$ of derivatized samples was injected into the GC/MS instrument, and splitless injection mode was used. A programmed column temperature was optimized to acquire a well separation. The temperatures of the injection port, the interface, and source temperature were set at $280^{\circ} \mathrm{C}$, $260^{\circ} \mathrm{C}$ and $230^{\circ} \mathrm{C}$, respectively. The measurements were made with electron impact ionization $(70 \mathrm{eV})$ in the full scan mode $(\mathrm{m} / z$ 30-550). The solvent post time was set to $5 \mathrm{~min}$. The GC-MS operating condition was the same as the previous experiment [15] except the column temperature program.

2.5. Data Analysis. Due to experimental variation and column aging, shifts in retention time between fingerprints may occur. When the total ion current chromatograms (TICs) were obtained, peak-alignment or warping techniques are commonly applied to compensate for minor shifts in retention times. Thus, in the subsequent data processing, the same variable manifested synchronous information in every 


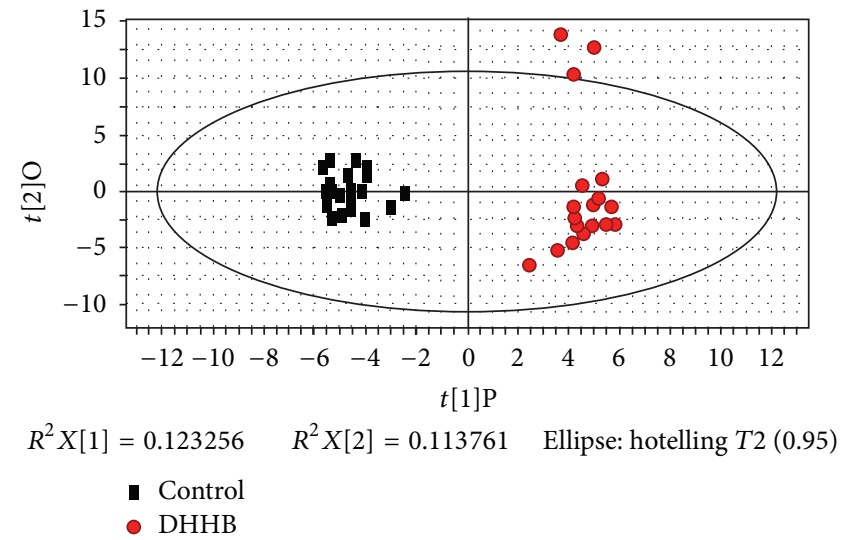

(a)

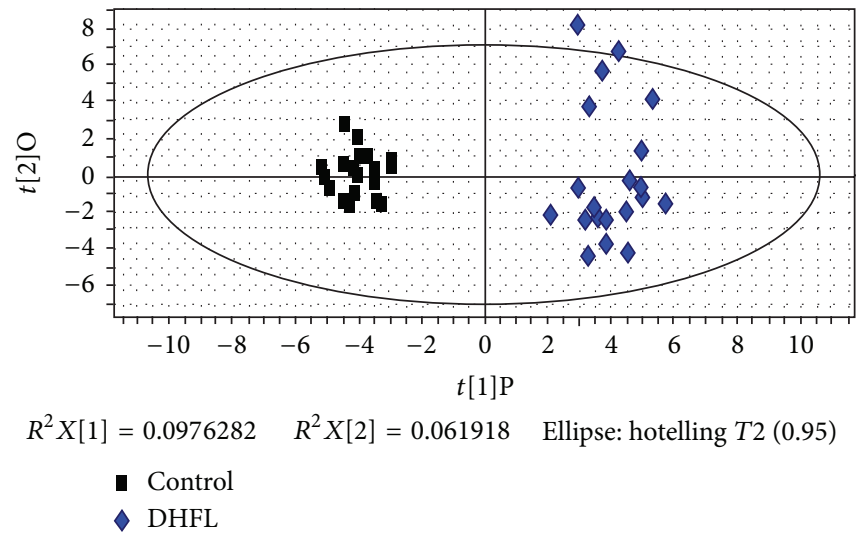

(b)

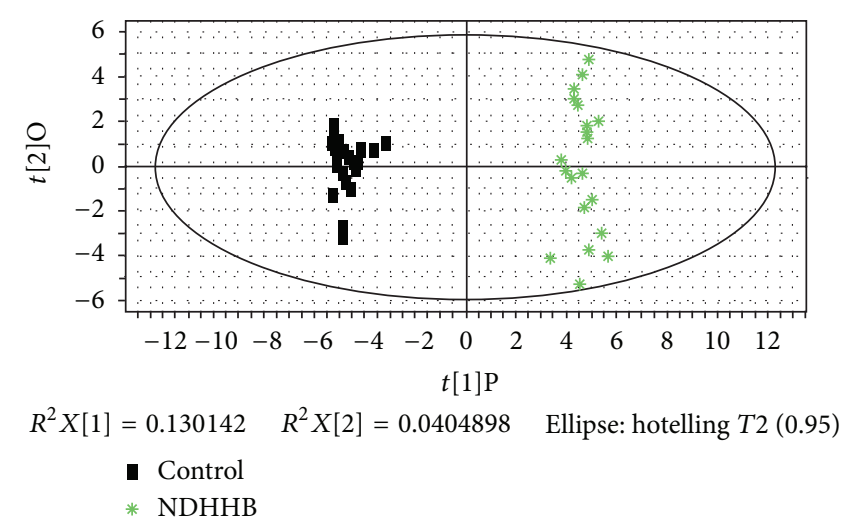

(c)

FIGURE 2: OPLS score plot of three syndromes compared to a healthy control group by symptoms and clinical indicators. (a) OPLS score plot of control and DHHB. (b) OPLS score plot of control and DHFL. (c) OPLS score plot of control and NDHHB.

profile. Therefore, all GC-MS raw files were converted to CDF format via the Agilent MSD Workstation software, and were subsequently processed by the XCMS toolbox (http:// metlin.scripps.edu/download/) using XCMS's default settings with the following exceptions: $x \mathrm{cmsSet}$ (full width at halfmaximum: fwhm $=5 ; \mathrm{S} / \mathrm{N}$ cutoff value: snthresh $=10$, $\max =25)$, group $(\mathrm{bw}=5)$. The resulting table (CSV file) was exported into Microsoft Excel (Microsoft Inc., USA) where normalization was performed prior to multivariate analyses. The resulting three-dimensional matrix involving peak index (RT- $m / z$ pair), sample names (observations), and normalized peak area percent was introduced into Simca-P 11.5 Umetrics software (Umea, Sweden) that was used for analysis of principal component analysis (PCA), partial least squares discriminant analysis (PLS-DA), and orthogonal partial least squares (OPLSs). Differential variables with VIP values [16] exceeding 1.5 between two different groups were generated from OPLS loadings plot. Subsequently, those variables were further analyzed by Mann-Whitney $U$-test to confirm the changes in metabolites by SPSS 17.0 (SPSS, Chicago, IL, USA) with the threshold $P$ value set at 0.1 . Firstly, the variables were identified by searching in NIST 2005 database. Then, standard compounds were used to confirm some of the identified metabolites.
Figure 1 shows a schematic diagram of the steps followed to determine the final list of potential biomarkers. The first step was to remove the differential information of $\mathrm{CHB}$ from the DHHB by removing the intersection of NDHHB and DHHB's differential information based on the ideas of the "same disease with different syndrome." The reduced set of first-step biomarkers were further filtered by taking advantage of the "different diseases with same syndrome." The final biomarkers were obtained from the intersection of the first-step DH biomarkers and biomarkers of DHFL.

\section{Results}

3.1. Establishment of the Potential Biomarkers of Clinical Symptoms and Indicators. All symptoms and clinical indicators were analyzed and utilized to distinguish the three syndrome groups (DHHB, NDHHB, and DHFL) and the healthy control group (control). Orthogonal partial least squares (OPLSs) was used to effectively extract variables responsible for the separation by removing variables unrelated to pathological status. Figures 2(a), 2(b), and 2(c) depict the OPLS score plots, which show that DHHB, NDHHB, and DHFL groups were clearly separated from the control group. The most meaningful characteristics were screened by OPLS loading 


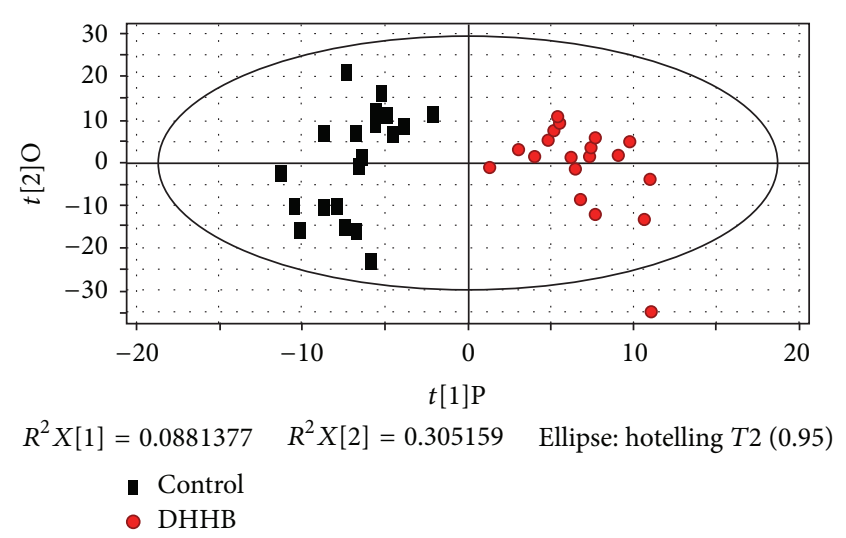

(a)

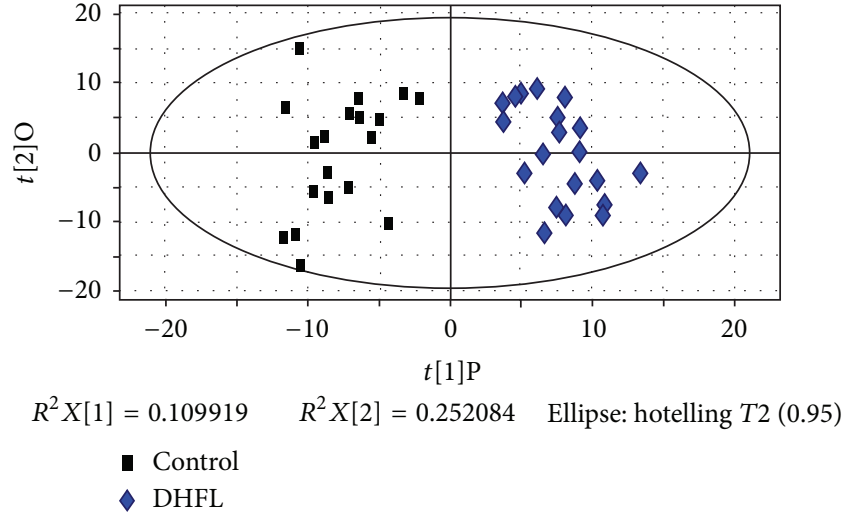

(b)

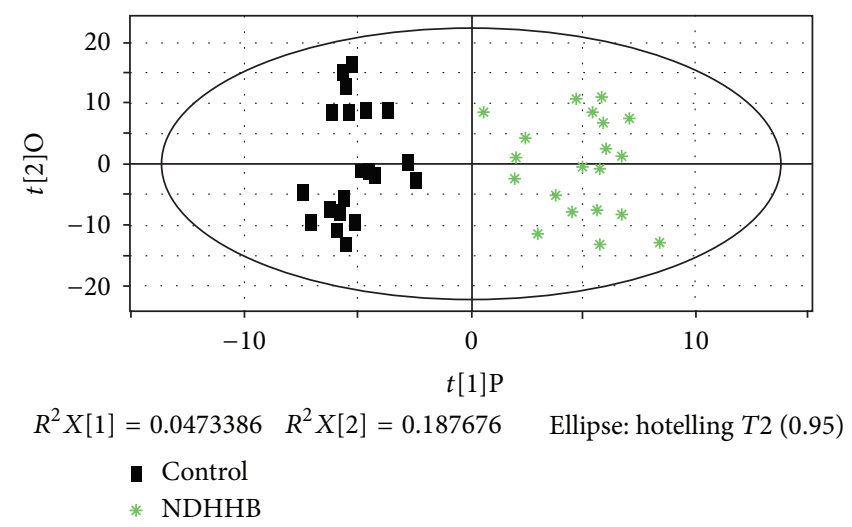

(c)

FIGURE 3: OPLS score plot of three syndromes compared to healthy control group by metabolites. (a) OPLS score plot of control and DHHB. (b) OPLS score plot of control and DHFL. (c) OPLS score plot of control and NDHHB.

plot analysis and are listed in Table 1 . The quality of the model was characterized by two performance statistics, $R^{2} Y$ (cum) and $Q^{2} Y$ (cum), indicating the total explanation and predictability of the model [17]. The information of models is summarized in Table 4.

\subsection{Establishment of the Potential Biomarkers of Urinary} Metabolic Profiles. Urine profiles obtained from GC-MS were analyzed for distinctions among the three syndromes and the control group by OPLS. Figures 3(a), 3(b) and 3(c) indicate the OPLS score plot, which show a clear separation for DHHB, NDHHB, and DHFL groups from the control group. The most important variables for the discriminative models were screened by loading plot analysis. The potential metabolic biomarkers of each syndrome differentiated from control group were identified by the NIST database and are summarized in Table 2. Model information is summarized in Table 4.

\subsection{Establishment of Potential Biomarkers of $\mathrm{DH}$ in $\mathrm{CHB}$ and} $F L$. Because groups of selected markers may contain information of syndrome and disease, the biomarkers of DH were further filtered. Thus the final set of potential biomarkers considered were those that remained after the intersection of $\mathrm{DHHB}$ and $\mathrm{NDHHB}$ was removed from $\mathrm{DHHB}$, and were intersected with DHFL. Figure 1 shows a schematic diagram of the steps. As to the former works, the potential macromicro biomarkers were obtained from the integration of differential metabolites, hierarchical corresponding symptoms and clinical indicators. The potential biomarkers are listed in Table 3.

3.4. Preliminary Verification of Identified Biomarkers. The potential biomarkers were verified in 55 blind test cases of $\mathrm{CHB}$ with two Syndromes (Dampness-Heat Syndrome (DH) and Non-Dampness-Heat Syndrome (NDH)) for Syndrome classification. Using only the potential biomarkers or only the clinical symptoms and indicators did not differentiate the two syndromes satisfactorily (Figures 4(a) and 4(b)); however, by including metabolites, symptoms, and clinical indicators in the analysis, resulted in a stronger differentiation (Figure 4(c)). It is worth mentioning that former classifications (in Sections 3.1 and 3.2) were performed by supervised OPLS, owing to the complexity of clinical samples. However, the $\mathrm{DH}$ could be classified from $\mathrm{NDH}$ by unsupervised PCA in this verification with the selected biomarkers, which revealed the strong ability of $\mathrm{DH}$ differentiation, though they need further verification in clinical. 
TABLE 1: Significantly different symptoms and clinical indicators identified in the three syndromes compared to a healthy control group.

\begin{tabular}{|c|c|c|c|c|}
\hline Indicators and symptoms & Group & $\mathrm{VIP}^{\mathrm{a}}$ & $P(\mathrm{M}-\mathrm{W})^{\mathrm{b}}$ & $\mathrm{FN}^{\mathrm{c}}$ \\
\hline Alkaline phosphatase & DHHB & 1.69 & 0.00 & +2.03 \\
\hline Apolipoprotein A-1 & DHHB & 1.74 & 0.00 & +1.91 \\
\hline Aspartate aminotransferase & DHHB & 1.62 & 0.00 & +2.77 \\
\hline Glutamyltransferase & DHHB & 1.65 & 0.00 & +2.06 \\
\hline Immunoglobulin G & DHHB & 2.01 & 0.00 & +2.06 \\
\hline Prealbumin & DHHB & 2.8 & 0.00 & -2.56 \\
\hline$\beta$-globin & DHHB & 2.52 & 0.00 & +2.67 \\
\hline Thick fur & DHHB & 1.81 & 0.00 & +1.73 \\
\hline Bitter taste & DHHB & 1.79 & 0.00 & +1.89 \\
\hline Slimy and curdy fur & DHHB & 1.75 & 0.00 & -1.73 \\
\hline Mean corpuscular hemoglobin concentration & DHHB & 1.84 & 0.00 & +2.15 \\
\hline Tongue color & DHHB & 2.15 & 0.00 & +1.96 \\
\hline Basophil & DHHB & 2.07 & 0.00 & +2.56 \\
\hline Fur color & DHHB & 2.68 & 0.00 & +2.58 \\
\hline String-like pulse & DHHB & 1.81 & 0.00 & +1.73 \\
\hline Alanine aminotransferase & DHFL & 2.08 & 0.00 & +2.16 \\
\hline Aspartate aminotransferase & DHFL & 1.78 & 0.00 & +1.82 \\
\hline Total cholesterol & DHFL & 2.10 & 0.00 & +1.87 \\
\hline Triglyceride & DHFL & 2.44 & 0.00 & +2.60 \\
\hline$\beta$-globin & DHFL & 2.54 & 0.00 & +2.31 \\
\hline Thick fur & DHFL & 1.92 & 0.00 & +1.64 \\
\hline Lack of strength & DHFL & 2.11 & 0.00 & +1.64 \\
\hline Dysphoria & DHFL & 1.98 & 0.00 & +1.67 \\
\hline Slimy and curdy fur & DHFL & 2.58 & 0.00 & -2.03 \\
\hline Uric acid & DHFL & 2.45 & 0.00 & +2.31 \\
\hline Glucose & DHFL & 1.95 & 0.00 & +1.92 \\
\hline Tongue color & DHFL & 2.03 & 0.00 & +1.68 \\
\hline Systolic pressure & DHFL & 1.89 & 0.00 & +2.02 \\
\hline Diastolic pressure & DHFL & 2.29 & 0.00 & +2.37 \\
\hline Fur color & DHFL & 3.08 & 0.00 & +2.58 \\
\hline Weight & DHFL & 2.79 & 0.00 & +2.44 \\
\hline String-like pulse & DHFL & 1.92 & 0.00 & +1.64 \\
\hline Alkaline phosphatase & NDHHB & 1.61 & 0.00 & +1.87 \\
\hline Apolipoprotein A-1 & NDHHB & 2.07 & 0.00 & +2.39 \\
\hline Activated partial thromboplastin time & NDHHB & 1.60 & 0.00 & +1.68 \\
\hline Hepatitis B core antibody & NDHHB & 2.85 & 0.00 & +2.90 \\
\hline Hepatitis B core antibody-immunoglobulin M & NDHHB & 1.76 & 0.00 & +2.90 \\
\hline Hepatitis B surface antigen & NDHHB & 3.04 & 0.00 & +2.90 \\
\hline Immunoglobulin G & NDHHB & 1.60 & 0.00 & +1.97 \\
\hline Prealbumin & NDHHB & 2.38 & 0.00 & -2.28 \\
\hline Triglyceride & NDHHB & 1.77 & 0.00 & +1.85 \\
\hline Total protein & NDHHB & 1.85 & 0.00 & +2.01 \\
\hline$\beta$-globin & NDHHB & 2.54 & 0.00 & +2.79 \\
\hline Teeth-marked tongue & NDHHB & 2.11 & 0.00 & +1.92 \\
\hline Gallbladder & NDHHB & 2.04 & 0.00 & +1.92 \\
\hline Relaxed pulse & NDHHB & 1.69 & 0.00 & +1.56 \\
\hline Lack of strength & NDHHB & 1.93 & 0.00 & +1.73 \\
\hline Mean corpuscular hemoglobin concentration & NDHHB & 1.79 & 0.00 & +2.11 \\
\hline Pre-S1 antibodies & NDHHB & 3.14 & 0.00 & +2.90 \\
\hline Pre-S1 antigen & NDHHB & 3.14 & 0.00 & +2.90 \\
\hline
\end{tabular}


TABLE 1: Continued.

\begin{tabular}{|c|c|c|c|c|}
\hline Indicators and symptoms & Group & $\mathrm{VIP}^{\mathrm{a}}$ & $P(\mathrm{M}-\mathrm{W})^{\mathrm{b}}$ & $\mathrm{FN}^{\mathrm{c}}$ \\
\hline Luxuriant or withered tongue & NDHHB & 1.73 & 0.00 & +1.73 \\
\hline Soggy pulse & NDHHB & 1.93 & 0.00 & +1.73 \\
\hline Basophil & NDHHB & 2.34 & 0.00 & +2.41 \\
\hline Diastolic pressure & NDHHB & 1.56 & 0.00 & +1.72 \\
\hline Mean platelet volume & $\mathrm{NDHHB}$ & 2.30 & 0.00 & +2.58 \\
\hline
\end{tabular}

${ }^{a}$ VIP: variable importance in the project.

${ }^{\mathrm{b}} P(\mathrm{M}-\mathrm{W})$ value was obtained from Mann-Whitney test (syndromes compared to healthy control).

${ }^{c} \mathrm{FN}$ is fold change of mean ranks calculated by the Mann-Whitney test (syndromes compared to healthy control). "+" means upregulated and "-" means downregulated.

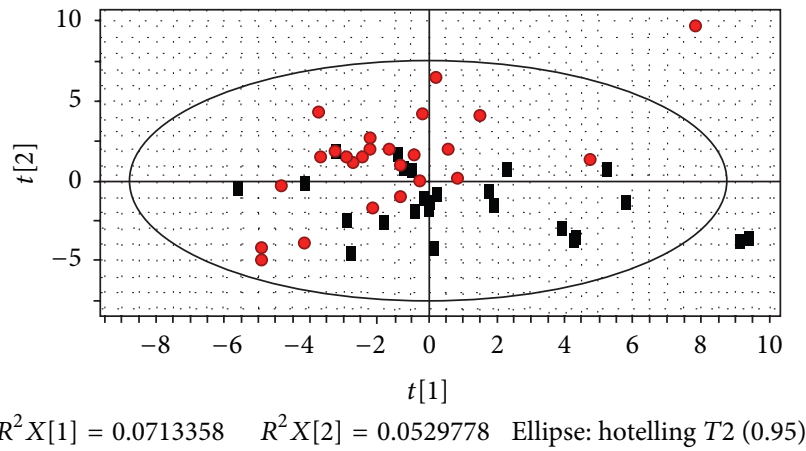

- DH syndrome

- $\mathrm{NDH}$ syndrome

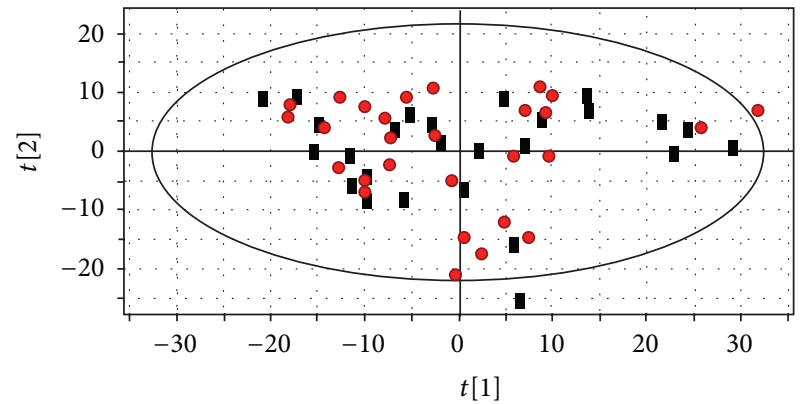

$R^{2} X[1]=0.2665115 \quad R^{2} X[2]=0.119742 \quad$ Ellipse: hotelling $T 2(0.95)$

- DH syndrome

- NDH syndrome

(a)

(b)

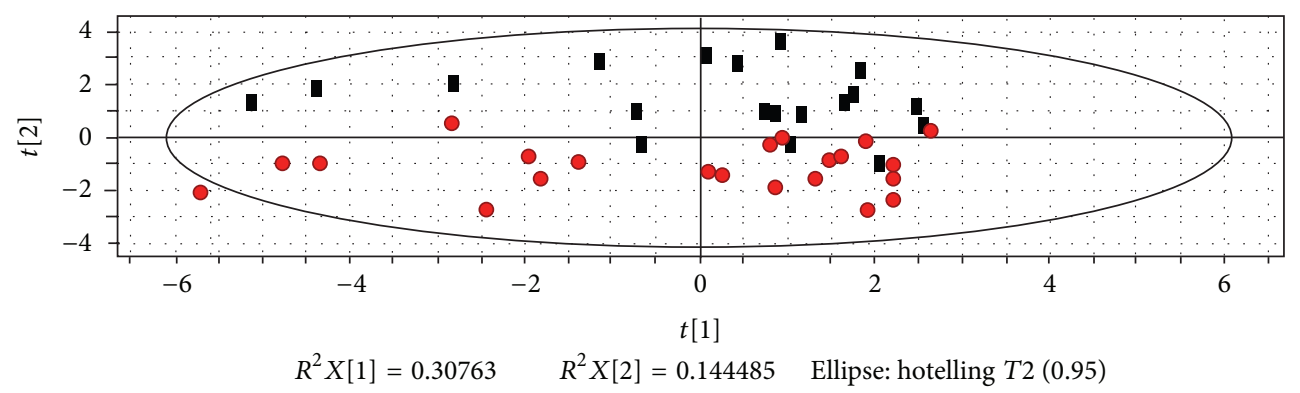

- DH syndrome

- $\mathrm{NDH}$ syndrome

(c)

FIGURE 4: PCA score plot of DH versus NDH with potential biomarkers. (a) PCA score plot of DH and NDH only by symptoms and clinical indicators. (b) PCA score plot of DH and NDH only by metabolites. (c) PCA score plot of DH and NDH by macro-micro biomarkers from the integration of differential metabolites, hierarchical corresponding symptoms and clinical indicators.

\section{Discussion}

In this study, we attempted to explore the macro-micro biomarkers of $\mathrm{DH}$, which could provide the feasibility and robustness for syndrome differentiation. The selected metabolites of DH were considered to be related with the pathogenesis. By analysis of KEGG (http://www.genome.jp/ kegg/), the 11 metabolic markers are related to biosynthesis of secondary metabolism, microbial metabolism in diverse environments, carbon fixation pathway in prokaryote, proteins digestion and absorption, and carbohydrate digestion and absorption, which could be classified in microbial metabolism and digestive capacity. These may correspond with "the disorder in transportation and transformation of the essence from food and drink" in TCM, which was regarded as one important reason for Dampness-Heat Syndrome $[18,19]$.

Aspartate transaminase (AST) is the only clinical indicator in our biomarkers. It may suggest that the clinical indicators are limited to classify the syndromes. But AST has been reported to be connected to $\mathrm{DH}$, with odds ratio (OR) value equal to 5.49 [20]. There is thus strong evidence that $\mathrm{DH}$ reflects inflammation of the liver damage. 
TABLE 2: Significantly different metabolites identified in the three syndromes compared to a healthy control group.

\begin{tabular}{|c|c|c|c|c|}
\hline Compound & Group & $\mathrm{VIP}^{\mathrm{a}}$ & $P(\mathrm{M}-\mathrm{W})^{\mathrm{b}}$ & $\mathrm{FN}^{\mathrm{c}}$ \\
\hline Acetic acid* & DHHB & 1.87 & 0.00 & -2.07 \\
\hline Succinic acid* & DHНB & 1.85 & 0.01 & -1.61 \\
\hline D-Xylose ${ }^{*}$ & DHHB & 2.22 & 0.00 & -1.96 \\
\hline Maltose & DHHB & 1.92 & 0.00 & +1.89 \\
\hline Butyrate $^{*}$ & DHHB & 1.87 & 0.00 & -1.72 \\
\hline Aminolevulinic acid ${ }^{*}$ & DHHB & 1.67 & 0.00 & -1.75 \\
\hline Ribitol & DHHB & 2.22 & 0.00 & -1.93 \\
\hline Creatinine $^{*}$ & DHHB & 1.77 & 0.01 & -1.63 \\
\hline Benzene* $^{*}$ & DHHB & 1.88 & 0.01 & -1.56 \\
\hline 2-Butenoic acid* & DHHB & 1.81 & 0.02 & -1.53 \\
\hline (R)-Mandelic acid* & DHНB & 1.68 & 0.01 & -1.59 \\
\hline Glutaconic acid* & DHHB & 1.68 & 0.02 & -1.54 \\
\hline Tartronic acid & DHНB & 2.28 & 0.00 & -2.10 \\
\hline Benzophenone & DHHB & 1.58 & 0.02 & -1.53 \\
\hline Pteridine & DHHB & 1.75 & 0.00 & -1.71 \\
\hline 3-Amino-1,2,4-triazole & DHHB & 1.74 & 0.02 & -1.51 \\
\hline 3-Indole butanoic acid* & DHНB & 1.61 & 0.02 & -1.50 \\
\hline 1-Cyclohexene carboxylic acid & DHHB & 1.93 & 0.01 & -1.66 \\
\hline 3-Indole acetic acid* & DHHB & 1.58 & 0.00 & -1.73 \\
\hline Pyrazinoic acid ${ }^{*}$ & DHHB & 1.62 & 0.00 & -2.00 \\
\hline Acetic acid* & DHFL & 1.52 & 0.00 & -1.70 \\
\hline Succinic acid* & DHFL & 1.90 & 0.00 & -1.81 \\
\hline D-Fructose & DHFL & 1.58 & 0.00 & -1.75 \\
\hline D-Galactose & DHFL & 1.69 & 0.00 & -1.99 \\
\hline Benzoic acid & DHFL & 1.51 & 0.01 & -1.59 \\
\hline Butyrate $^{*}$ & DHFL & 1.54 & 0.01 & -1.65 \\
\hline D-Gluconic acid & DHFL & 1.51 & 0.00 & -1.70 \\
\hline Amino levulinic acid* & DHFL & 1.54 & 0.00 & -1.82 \\
\hline Glutarate & DHFL & 1.57 & 0.01 & -1.61 \\
\hline Creatinine $^{*}$ & DHFL & 1.65 & 0.00 & -1.69 \\
\hline Gulonic acid & DHFL & 1.65 & 0.00 & -1.72 \\
\hline Glucaric acid & DHFL & 1.63 & 0.00 & -2.02 \\
\hline 3-Indole acetic acid* & DHFL & 1.72 & 0.00 & -2.09 \\
\hline (R)-Mandelic acid* & DHFL & 1.76 & 0.00 & -1.75 \\
\hline 3-Indole butanoic acid* & DHFL & 1.68 & 0.00 & -1.68 \\
\hline 1-Cyclohexenecarboxylic acid & DHFL & 1.86 & 0.00 & -1.81 \\
\hline Pseudouridine & DHFL & 1.79 & 0.00 & -2.10 \\
\hline Glutaconic acid ${ }^{*}$ & DHFL & 1.77 & 0.00 & -1.71 \\
\hline Tetradecanoic acid & DHFL & 1.68 & 0.00 & -1.74 \\
\hline Ethylene & DHFL & 1.51 & 0.01 & -1.57 \\
\hline Pteridine & DHFL & 1.57 & 0.00 & -1.73 \\
\hline Pyrazinoic acid ${ }^{*}$ & DHFL & 1.53 & 0.00 & -2.19 \\
\hline $\begin{array}{l}\text { 1-(1-Benzyl-1H-indol-3-yl)- } \\
\text { 2,2,2-trifluoroethanone }\end{array}$ & DHFL & 1.63 & 0.00 & -1.83 \\
\hline Glycine* & NDHHB & 2.11 & 0.01 & +1.62 \\
\hline D-Xylose* & NDHHB & 2.15 & 0.01 & -1.57 \\
\hline D-Gluconic acid & NDHHB & 1.87 & 0.00 & +1.85 \\
\hline Ribitol & NDHHB & 2.19 & 0.01 & -1.57 \\
\hline 2,3-Butanedione & NDHHB & 1.89 & 0.02 & +1.56 \\
\hline Tartronic acid & NDHHB & 2.21 & 0.00 & -1.66 \\
\hline Vanillylmandelic acid & NDHHB & 1.94 & 0.05 & +1.42 \\
\hline 3-Amino-1,2,4-triazole & NDHHB & 2.31 & 0.03 & -1.48 \\
\hline
\end{tabular}


TABLE 3: List of the macro-micro biomarkers of $\mathrm{DH}$ in $\mathrm{CHB}$ and NFL.

\begin{tabular}{lc}
\hline Biomarkers & Category \\
\hline (R)-mandelic acid & Metabolites \\
1-Cyclohexenecarboxylic acid & Metabolites \\
3-Indole acetic acid & Metabolites \\
3-Indole butanoic acid & Metabolites \\
Acetic acid & Metabolites \\
Amino levulinic acid & Metabolites \\
Butyrate & Metabolites \\
Creatinine & Metabolites \\
Glutaconic acid & Metabolites \\
Pteridine & Metabolites \\
Pyrazinoic acid & Metabolites \\
Succinic acid & Metabolites \\
Aspartate aminotransferase & Indicators \\
Thick fur & Symptoms \\
Slimy and curdy fur & Symptoms \\
Tongue color & Symptoms \\
Fur color & Symptoms \\
String-like pulse & Symptoms \\
\hline
\end{tabular}

TABLE 4: Summary of the modeling quality of OPLS analysis.

\begin{tabular}{lcccc}
\hline Name & $\mathrm{No}^{\mathrm{a}}$ & $R^{2} X_{\text {cum }}{ }^{\mathrm{b}}$ & $R^{2} Y_{\text {cum }}{ }^{\mathrm{c}}$ & $Q^{2} Y_{\text {cum }}{ }^{\mathrm{d}}$ \\
\hline $1 \mathrm{~A}$ & $1 \mathrm{P}+1 \mathrm{O}^{\mathrm{e}}$ & 0.24 & 0.97 & 0.91 \\
$1 \mathrm{~B}$ & $1 \mathrm{P}+1 \mathrm{O}$ & 0.16 & 0.96 & 0.78 \\
$1 \mathrm{C}$ & $1 \mathrm{P}+1 \mathrm{O}$ & 0.17 & 0.98 & 0.93 \\
$2 \mathrm{~A}$ & $1 \mathrm{P}+2 \mathrm{O}$ & 0.50 & 0.89 & 0.70 \\
$2 \mathrm{~B}$ & $1 \mathrm{P}+3 \mathrm{O}$ & 0.56 & 0.90 & 0.48 \\
2C & $1 \mathrm{P}+3 \mathrm{O}$ & 0.49 & 0.91 & 0.57
\end{tabular}

${ }^{\mathrm{a}}$ No represents the number of components.

${ }^{\mathrm{b}, \mathrm{c}} R^{2} X_{\text {cum }}$ and $R^{2} Y_{\text {cum }}$ represent the cumulative sum of squares (SSs) of all the $X$ 's and $Y$ 's explained by all extracted components.

${ }^{\mathrm{d}} \mathrm{Q}^{2} Y_{\text {cum }}$ is an estimate of how well the model predicts the $Y$ 's.

${ }^{\mathrm{e}} 1 \mathrm{P}+1 \mathrm{O}$ : one predictive component and one orthogonal component for establishing the OPLS model.

Tongue diagnosis is of great importance for syndrome differentiation in TCM, determining the treating principle, prescribing a formula, and predicting the prognosis [21]. Except string-like pulse, other differential symptoms are the characterization of tongue, which is one of the direct objective bases for TCM clinical diagnosis and treatment. In our opinion, pulse diagnosis is as important as tongue diagnosis, so ultimately a more comprehensive analysis for the combination of them is needed.

Although only metabonomics was utilized in this study, we suggest that it would be valuable to expand beyond Metabonomics to system biology owing to the similarity between the various omics. Including a full System Biology approach to the determination of informative biomarkers will provide a more comprehensive and accurate syndrome differentiation. We thus suggest that genes, proteins, metabolites, and clinical information should all be integrated in future analyses.

\section{Conclusion}

This study is the first time that biomarkers of $\mathrm{DH}$ were obtained by a macro-micro approach with the integration of omic and clinical information to provide an effective and objective and repeatable approach for Chinese personalized medicine. Moreover, the preliminary verification indicated the feasibility and robustness of the approach for dampnessheat syndrome differentiation. Thus, these DH biomarkers could be used to provide a foundation on which we can to develop a possible population-screening tool for selecting target individuals and for creating an evaluation index for personalized treatment based on syndrome differentiation.

\section{Authors' Contribution}

Jianye Dai, Shujun Sun, and Jinghua Peng contributed equally to this work and should be considered cofirst authors.

\section{Acknowledgments}

This work was supported by the National Science and Technology Major Project (2012ZX10005001-004 and 2012ZX09303009-001), Shanghai Interdisciplinary Cultivation Platform of Outstanding and Innovative Postgraduates, and Shanghai "085" Science, and Technology Innovation Supporting Project for Top-grade Discipline Construction.

\section{References}

[1] Y. F. Liaw, N. Leung, J. H. Kao et al., "Asian-Pacific consensus statement on the management of chronic hepatitis B: a 2008 update," Hepatology International, vol. 2, no. 3, pp. 263-283, 2008.

[2] H. Y. Wang, H. M. Li, B. Yang, and J. J. Xu, “The relationship between distribution characteristics of TCM constitution and Syndromes in 141 patients with fatty liver," Journal of Beijing University of TCM, vol. 33, no. 7, pp. 500-502, 2010.

[3] J. Chang, X. F. Pan, W. W. Qiu, G. Y. Yang, and L. Q. Wang, "Objectivized study on syndrome differentiation for chronic hepatitis B," Jiangsu Journal of TCM, vol. 27, no. 5, pp. 26-27, 2006.

[4] J. L. Tang, B. Y. Liu, and K. W. Ma, "Traditional Chinese medicine," The Lancet, vol. 372, no. 9654, pp. 1938-1940, 2008.

[5] W. Zhang, T. Leonard, F. Bath-Hextall et al., "Chinese herbal medicine for atopic eczema," Cochrane Database of Systematic Reviews, no. 2, Article ID CD002291, 2005.

[6] J. M. Ezzo, M. A. Richardson, A. Vickers et al., "Acupuncturepoint stimulation for chemotherapy-induced nausea or vomiting," Cochrane Database of Systematic Reviews, no. 2, Article ID CD002285, 2006.

[7] M. H. Pittler and E. Ernst, "Artemether for severe malaria: a meta-analysis of randomized clinical trials," Clinical Infectious Diseases, vol. 28, no. 3, pp. 597-601, 1999.

[8] M. A. Hamburg and F. S. Collins, "The path to personalized medicine," The New England Journal of Medicine, vol. 363, no. 4, pp. 301-304, 2010.

[9] J. L. Yuan, H. Zhang, L. Wang et al., "Biochemical characteristics of traditional Chinese medicine syndromes and their elements 
in patients with hepatitis B cirrhosis," Journal of Chinese Integrative Medicine, vol. 9, no. 4, pp. 374-381, 2011.

[10] L. H. Zhao, C. Xiao, X. P. Yan et al., "Correlation between heat or cold syndrome and cytokine, and laboratory index in women with early rheumatoid arthritis," Acta Universitatis Traditionis Medicalis Sinensis Pharmacologiaeque Shanghai, vol. 20, no. 1, pp. 21-24, 2006.

[11] X. Q. Yue and Q. Liu, "Analysis of studies on pattern recognition of tongue image in traditional Chinese medicine by computer technology," Journal of Chinese Integrative Medicine, vol. 2, no. 5, pp. 326-329, 2004.

[12] B. Pang, D. Zhang, N. Li, and K. Wang, "Computerized tongue diagnosis based on Bayesian networks," IEEE Transactions on Biomedical Engineering, vol. 51, no. 10, pp. 1803-1810, 2004.

[13] Chinese Society of Hepatology and Chinese Society of Infectious Diseases, Chinese Medical Association, "The guideline of prevention and treatment for chronic hepatitis B, (2010 version)," Chinese Journal of Hepatology, vol. 19, no. 1, pp. 1324, 2011.

[14] F. Jian-gao and Chinese Liver Disease Association, "Guidelines for management of nonalcoholic fatty liver disease: an updated and revised edition," Chinese Journal of Hepatology, vol. 18, no. 3, pp. 163-166, 2010.

[15] S. J. Sun, J. Y. Dai, W. Y. Wang et al., "Metabonomic evaluation of ZHENG differentiation and treatment by fuzhenghuayu tablet in hepatitis-B-caused cirrhosis," Evidence-Based Complementary and Alternative Medicine, vol. 2012, Article ID 453503, 8 pages, 2012.

[16] L. Eriksson, E. Johansson, and N. Kettaneh-Wold, Multiand Megavariate Data Analysis, Part 1: Basic Principles and Applications, Umetrics AB, Umeå, Sweden, 2nd edition, 2001.

[17] P. Yin, D. Wan, C. Zhao et al., "A metabonomic study of hepatitis B-induced liver cirrhosis and hepatocellular carcinoma by using RP-LC and HILIC coupled with mass spectrometry," Molecular BioSystems, vol. 5, no. 8, pp. 868-876, 2009.

[18] J. B. Zhang, "Complete Works of Jingyue (jing yue quan shu)," 1640.

[19] T. S. Ye, “Treatise on Worm Heat Disease (wen bing lun)," 1746.

[20] N. J. Li, J. Wang, and S. K. Yao, "Relationship between Dampness-Heat Syndrome and liver inflammation indicators," Chinese Journal of Basic Medicine in Traditional Chinese Medicine, vol. 17, no. 3, pp. 294-295, 2011.

[21] T. F. Wang, Diagnostics of Traditional Chinese Medicine, People's Medical Publishing House, Beijing, China, 2nd edition, 2007. 


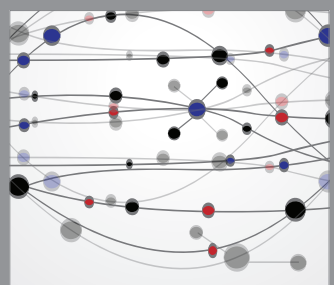

The Scientific World Journal
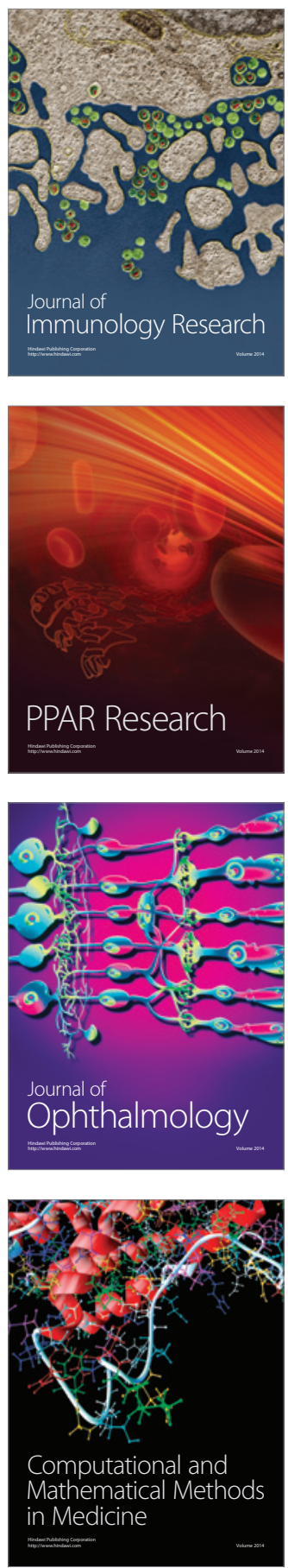

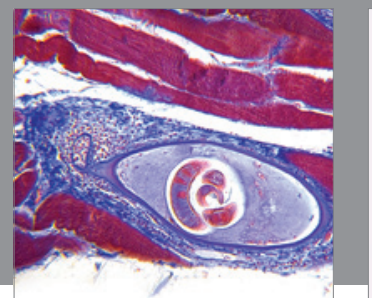

Gastroenterology

Research and Practice
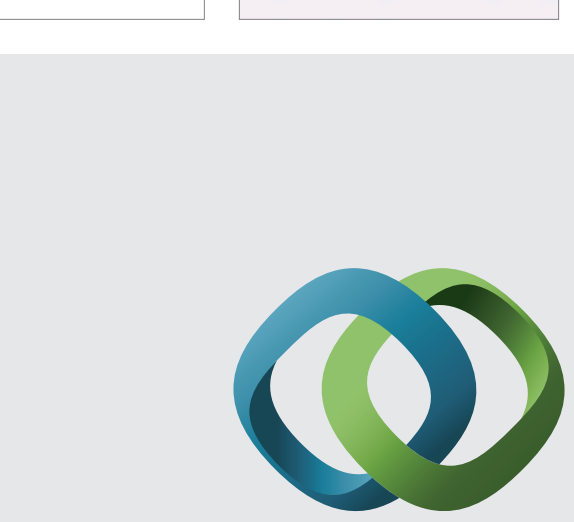

\section{Hindawi}

Submit your manuscripts at

http://www.hindawi.com
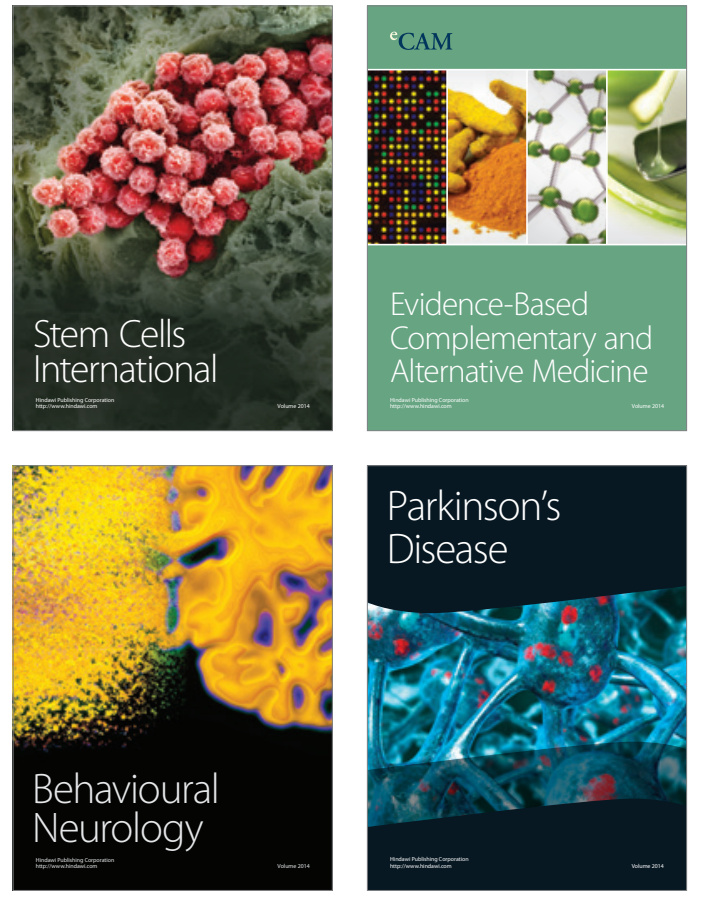
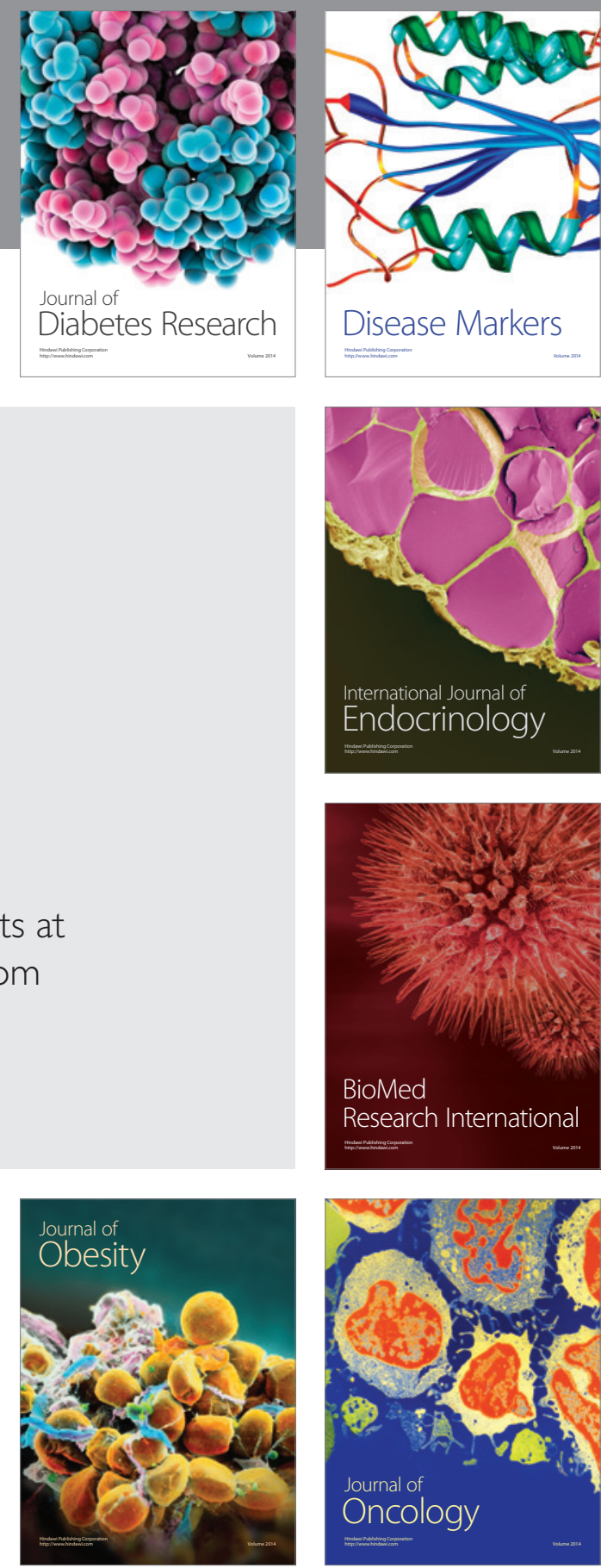

Disease Markers
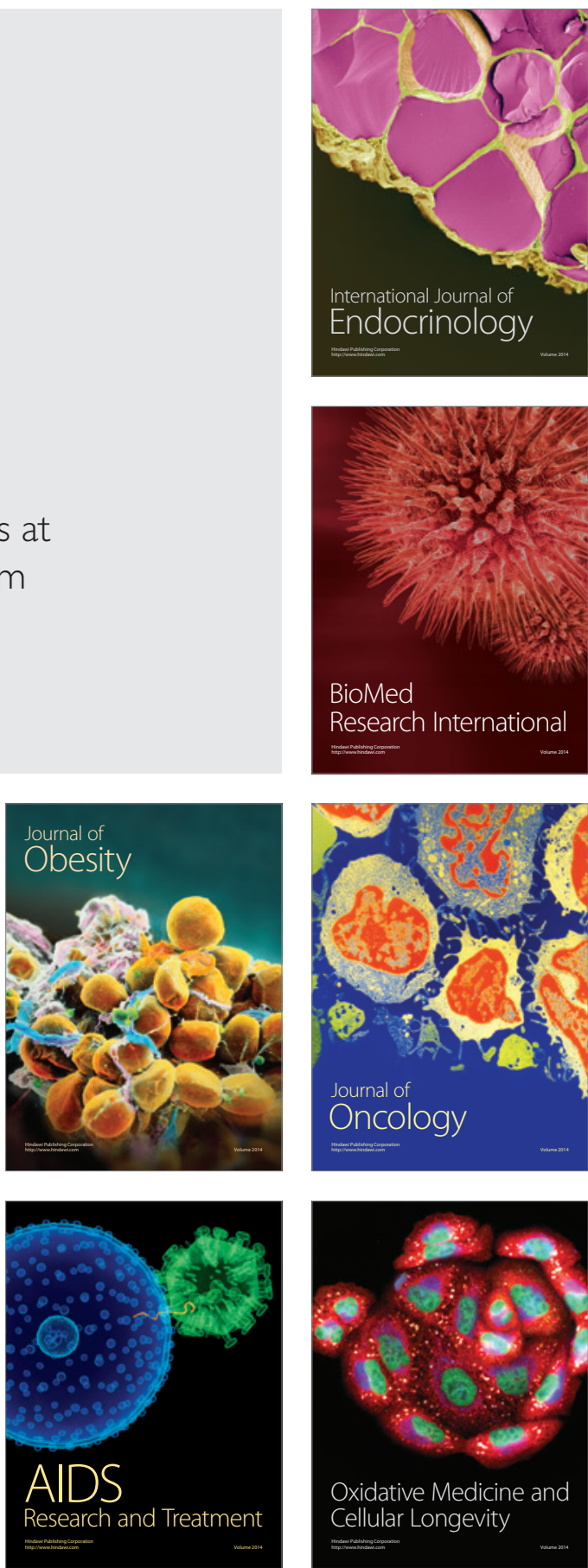